\title{
Application of Probe Electrospray Ionization Mass Spectrometry (PESI-MS) to Clinical Diagnosis: Solvent Effect on Lipid Analysis
}

\author{
Mridul Kanti Mandal, ${ }^{1}$ Kentaro Yoshimura, ${ }^{2}$ Lee Chuin Chen, ${ }^{3}$ Zhan Yu, ${ }^{4}$ \\ Tadao Nakazawa, ${ }^{5}$ Ryohei Katoh, ${ }^{5}$ Hideki Fujii, ${ }^{6}$ Sen Takeda, ${ }^{2}$ Hiroshi Nonami, ${ }^{7}$ \\ Kenzo Hiraoka ${ }^{1}$ \\ ${ }^{1}$ Clean Energy Research Center, University of Yamanashi, Kofu, Yamanashi, Japan \\ ${ }^{2}$ Department of Anatomy and Cell Biology, Interdisciplinary Graduate School of Medicine and Engineering, \\ University of Yamanashi, Yamanashi, Japan \\ ${ }^{3}$ Interdisciplinary Graduate School of Medicine and Engineering, University of Yamanashi, Yamanashi, Japan \\ ${ }^{4}$ College of Chemistry and Biology, Shenyang Normal University, Shenyang, China \\ ${ }^{5}$ Department of Human Pathology, Interdisciplinary Graduate School of Medicine and Engineering, University of Yamanashi, \\ Yamanashi, Japan \\ ${ }^{6}$ Department of Surgery, Interdisciplinary Graduate School of Medicine and Engineering, University of Yamanashi, \\ Yamanashi, Japan \\ ${ }^{7}$ Plant Biophysics/Biochemistry Research Laboratory, Faculty of Agriculture, Ehime University, Matsuyama, Japan
}

\begin{abstract}
We have examined several combinations of solvents with the aim of optimizing the ionization conditions for molecular diagnosis of malignant tumours by PESI-MS. Although the best conditions may depend on the actual species in the sample, the optimal conditions for renal cell carcinoma (RCC) were achieved by using alcohols. PESI-MS successfully delineated the differential expression of phospholipids (PCs) and triacylglycerols (TAGs) in noncancerous and RCC tissues by using these solvent systems. This study paves the way for the application of PESI-MS in medical samples.
\end{abstract}

Key words: Electrospray, Probe Electrospray, Pathology, Biological Specimen, Diagnostics

\section{Introduction}

$\mathrm{T}$ he application of mass spectrometry (MS) to medicine has a relatively long history, dating back to 1980 s when gas chromatography mass spectrometry (GC-MS) had been applied in the kinetic analysis of bile acid in patients with Crohn's disease [1]. Subsequently, the development of new ionization techniques such as electrospray ionization (ESI)

Electronic supplementary material The online version of this article (doi:10.1007/s13361-012-0462-3) contains supplementary material, which is available to authorized users.

Correspondence to: Kenzo Hiraoka; e-mail: hiraoka@yamanashi.ac.jp, Sen Takeda; e-mail: stakeda@yamanashi.ac.jp
[2] and matrix-assisted laser desorption ionization (MALDI) [3] have accelerated the application of MS in medicine [4]. Recently, desorption electrospray ionization mass spectrometry (DESI-MS) has been applied successfully to lipid characterization [5] and biological tissue imaging [6, 7]. Takáts et al. reported a promising technique named "rapid evaporative ionization mass spectrometry (REI-MS)," which allows realtime identification of tissue properties during surgery [8].

To address clinical requirements, we have developed probe electrospray ionization (PESI) [9, 10], which uses a solid needle as a sampling probe and an ESI emitter. PESI-MS has been applied to the direct analysis of biological samples, and was validated as a useful method for measuring mass spectra without 


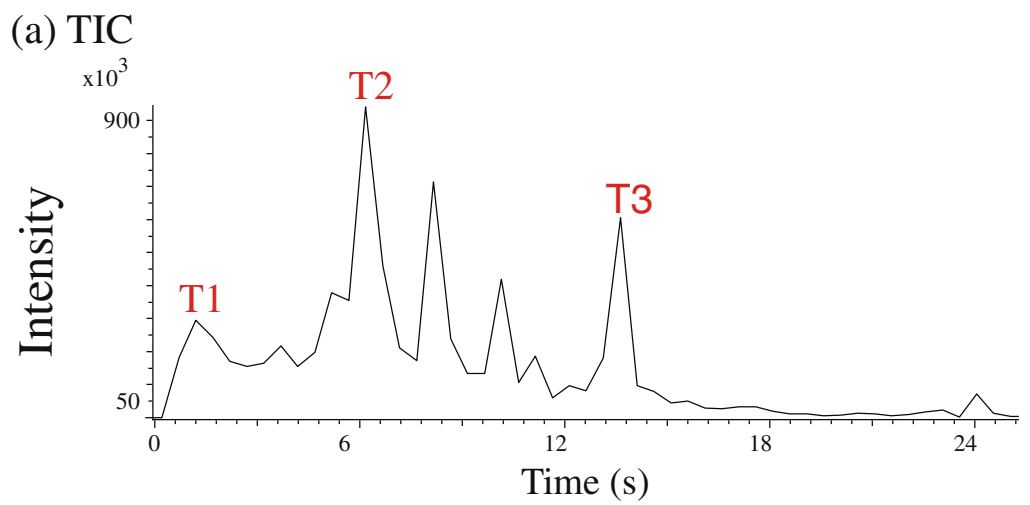

(b) MS (T1)

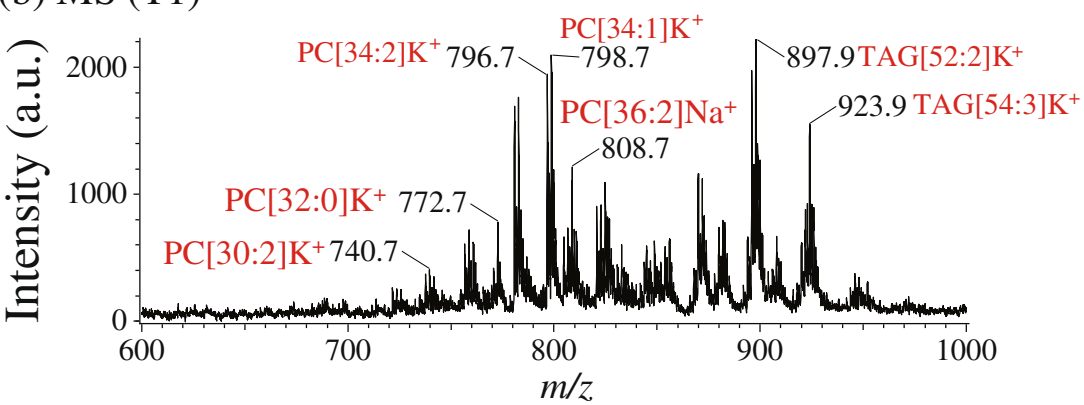

(c) MS (T3)

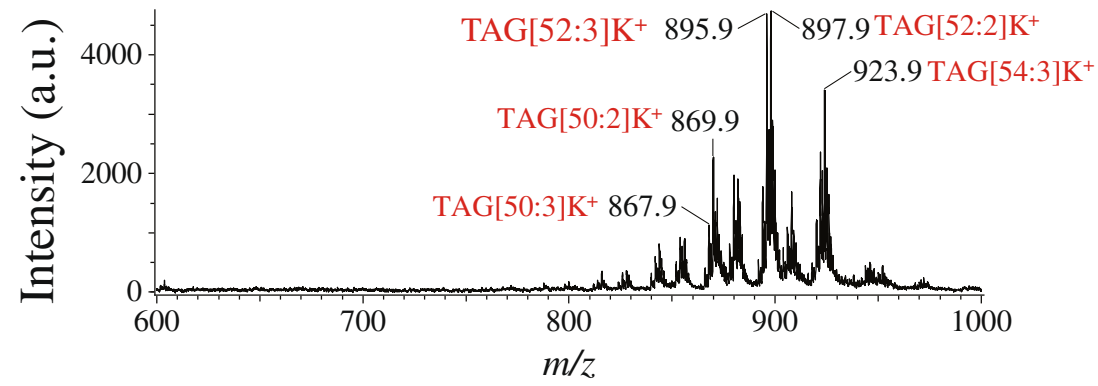

Figure 1. Single shot PESI mass spectra for renal cancer tissue using $\mathrm{EtOH} / \mathrm{H}_{2} \mathrm{O}(1 / 1[\mathrm{vol} / \mathrm{vol}])$ as solvent. (a) Total ion chromatogram (TIC); (b) mass spectrum measured at $\mathrm{T} 1$; (c) mass spectrum measured at $\mathrm{T} 3$ which is similar to that measured at $\mathrm{T} 2$

any sample pretreatment [11, 12]. Recently, we have also applied PESI-MS to real-time analysis of organs in living mice, and showed the usefulness in distinguishing differences in lipid composition of hepatocytes between normal and steatotic mice. All mice used in this study tolerated the experimental procedure of PESI-MS because of its low invasiveness, and survived for months until being sacrificed for further analyses [13].

In our previous work [10], it was found that a single-shot PESIMS using a $0.5 \mathrm{~mm}$-diameter titanium (Ti) wire could virtually ionize all the analytes contained in a droplet at the needle tip. The analytes undergo sequential and exhaustive ionization according to their surface activities, and these phenomena help alleviate the ion-suppression effect that is inherent to capillary-based ESI, in which sample solution is continuously supplied to the capillary.

To examine the solubility of lipids, we have used water and several organic solvents for lipids extraction in human cancer tissues. Under these conditions, renal cell carcinoma (RCC) is characterized by accumulation of triacylglycerol (TAG) $[14,15]$, which is a good model for proving the usefulness of PESI-MS.

\section{Experimental}

\section{Probe Preparation}

The probe preparation procedures were basically similar to those described in earlier works $[10,16]$.

Table 1. PESI Solvents for Extraction and Separation of Lipids with Reference Figures

\begin{tabular}{|c|c|c|c|}
\hline PESI solvents & Molecular species & $\begin{array}{l}\text { Extraction and } \\
\text { separation }\end{array}$ & $\begin{array}{l}\text { Reference } \\
\text { figure }\end{array}$ \\
\hline Ethanol/ $\mathrm{H}_{2} \mathrm{O}$ & $\mathrm{CE}, \mathrm{PC}$ and $\mathrm{TAG}$ & Good & Fig. 1 \\
\hline Isopropanol/ $\mathrm{H}_{2} \mathrm{O}$ & $\mathrm{CE}, \mathrm{PC}$, and TAG & Good & Fig. S2 \\
\hline Acetonitrile $/ \mathrm{H}_{2} \mathrm{O}$ & $\mathrm{CE}, \mathrm{PC}$, and $\mathrm{TAG}$ & Poor & Fig. S3 \\
\hline Methanol/ $\mathrm{H}_{2} \mathrm{O}$ & $\mathrm{CE}, \mathrm{PC}$, and TAG & Good & Fig. S4 \\
\hline n-Propanol & $\mathrm{CE}, \mathrm{PC}$, and $\mathrm{TAG}$ & Good & Fig. S5 \\
\hline Tetrahydrofuran $/ \mathrm{H}_{2} \mathrm{O}$ & $\mathrm{CE}, \mathrm{PC}$, and TAG & Poor & Fig. S6 \\
\hline
\end{tabular}




\section{Probe Electrospray Ionization (PESI)}

A schematic representation of the experimental system is shown in Supplementary Figure 1 [10]. PESI mass spectra were obtained with a time-of-flight mass spectrometer (AccuTOF; JEOL, Tokyo, Japan). In this study, PESI-MS was operated using single and continuous shot modes $[9,10]$. For single-shot probe electrospray, a surface-modified Ti wire with a diameter of $0.5 \mathrm{~mm}$ was used as the sampling probe. The droplet volume trapped at the probe tip was about $50 \mathrm{~nL}$ [10]. The droplet was left to be electrosprayed completely until the electrospray current decreased to zero.

For continuous shot probe electrospray, a Ti wire with a diameter of $0.1 \mathrm{~mm}$ was used. The droplet volume trapped at the needle tip was about a few $\mathrm{pL}$ [11]. The needle tip was periodically renewed by sampling with sampling frequency of $3 \mathrm{~Hz}$.

For both the single and continuous shot modes of operation, a linear actuator (Citizen, Chiba, Japan) was used to drive the Ti needle along a vertical axis perpendicular to the apex of the ion sampling orifice of the mass spectrometer. The stroke distance of the wire was $12 \mathrm{~mm}$. The invasion depth of the needle into the sample was $0.5 \mathrm{~mm}$, which was regulated as described previously [10]. With this invasion depth, the spatial resolutions of the single and continuous shot modes were about 0.5 and $0.1 \mathrm{~mm}$, respectively. When the wire with sample droplet on its tip was moved to the highest position, a high voltage of 3$3.5 \mathrm{kV}$ was applied to generate electrospray. The electrospray duration for the single and continuous shot PESI was $\sim 20 \mathrm{~s}$ and a few hundred ms, respectively, depending on the experimental conditions (i.e., solvents used, surface conditions of the renal tissue, etc.). Three consecutive single-shot PESI experiments were performed that confirmed excellent reproducibility. Exact mass analysis for identification of observed peaks was performed with a high-resolution mass spectrometer (Bench Top Orbitrap Exactive; Thermo, Bremen, Germany).

\section{Sample Preparation}

Solvents and buffer solutions were high-performance liquid chromatography (HPLC) grade (Sigma, Tokyo, Japan), and were used without further purification. Pure water was prepared using a Milli-Q system (Millipore, Bedford, MA, USA). Cancer tissues from patients diagnosed as RCC and their adjacent noncancer counterparts were obtained from the Department of Surgery, Faculty of Medicine, University of Yamanashi, and were handled according to university regulations. The size of kidney tissue was about $10 \times 4 \times 2 \mathrm{~mm}^{3}$. The tissues were stored at $-80{ }^{\circ} \mathrm{C}$, thawed, and used for measurements. All the procedures relating to the human specimens were approved by the Ethical Committee of the University of Yamanashi Faculty of Medicine.

\section{Solvent Selection for Lipid Analysis}

Mixtures of organic solvents and $\mathrm{H}_{2} \mathrm{O}$ with relative concentrations of $0 \%, 25 \%, 50 \%, 75 \%$, and $100 \%$ were used for lipids extraction from the kidney tissues. Pure water gave comparatively weak ion signals, possibly because of less efficient lipids extraction. In contrast, pure organic solvents generally gave stronger ion signals than water, whereas mass spectra were less reproducible because of the rapid evaporation of the droplet trapped on the needle tip. Shanta et al. recently reported that a binary matrix has superior performance to conventional matrix during lipidomic-based MALDI imaging [17]. We found that a binary solvent system with a mixing ratio of $1 / 1$ (vol/vol) gave better results among all the solvent systems examined. Thus, data obtained by binary solvents with mixing ratio of organic solvents $/ \mathrm{H}_{2} \mathrm{O}(1 / 1[\mathrm{vol} / \mathrm{vol}])$ are presented as representative mass spectra.

Single-shot PESI mass spectra were obtained immediately after dropping $1 \mu \mathrm{L}$ of binary solvents on the surface of the kidney tissue, which created on the tissue a spot size with a diameter of less than $2 \mathrm{~mm}$. We referred to previous efforts on profiling and imaging of tissue by MALDI-MS [18, 19] to select ideal solvents for extracting lipid components from the specimens. In these studies, alcohol was employed to remove salts, lipids, and any other component that would interfere with spectral analyses of biological specimens [18, 19]. Additionally, Seeley et al. also reported that alcohol-based washes, in particular isopropanol, are the most effective for protein analysis in a MALDI imaging as they effectively remove lipids from biological specimens [20]. Therefore, using alcohols seemed to be one of the ideal choices for better extraction of molecules, especially lipids that are major constituents of cell membranes.

\section{Results and Discussion}

$\mathrm{EtOH} / \mathrm{H}_{2} \mathrm{O}$ was found to be a suitable binary solvent for lipids extraction from renal tissues. Figure 1 displays the total ion chromatogram (TIC) and the PESI mass spectra at points T1 and T3. At T1, the onset of electrospray, sodiated and potassiated phosphatidylcholine PCs as major ions and potassiated TAGs as minor ions are observed. At T2 and T3, potassiated TAGs are observed with almost no ion signals from PCs. The observed sequential ionization of PCs and TAGs in Fig. 1 is attributed to the fact that analytes that are preferentially enriched on the surface of the liquid droplet (PCs) are electrosprayed first and those being suppressed (TAGs) are electrosprayed after exhaustion of surfaceenriched analytes (PCs) [10]. This explains why the suppression effect is minimized in PESI.

In addition to EtOH/ $\mathrm{H}_{2} \mathrm{O}$, we have also examined binary solutions of isopropanol $/ \mathrm{H}_{2} \mathrm{O}, \mathrm{CH}_{3} \mathrm{CN} / \mathrm{H}_{2} \mathrm{O}, \mathrm{MeOH} / \mathrm{H}_{2} \mathrm{O}$, n-Propanol $/ \mathrm{H}_{2} \mathrm{O}$ and $\mathrm{THF} / \mathrm{H}_{2} \mathrm{O}$ for single shot PESI experiments (see Supplementary Figures S2, S3, S4, S5, and S6). When $\mathrm{CH}_{3} \mathrm{CN} / \mathrm{H}_{2} \mathrm{O}$ was used in single-shot PESI (Supplementary Figure S3), no separation was observed between PCs and TAGs. The weaker intensities of TAGs compared with those of PCs may result from decreased extraction efficiency of TAG in $\mathrm{CH}_{3} \mathrm{CN} / \mathrm{H}_{2} \mathrm{O}$, than in alcohols $/ \mathrm{H}_{2} \mathrm{O}$ systems.

Finally, we tested $\mathrm{THF} / \mathrm{H}_{2} \mathrm{O}(1 / 1[\mathrm{vol} / \mathrm{vol}])$ as a solvent [21]. As shown in Supplementary Figure S6, the extraction efficiency for TAGs from RCC is much poorer than that for 
alcohol-based solvent systems. The results obtained by using various solvent systems for cancerous tissue analysis are summarized in Table 1.

Similar experiments were also performed to assure the low concentration of TAG in noncancerous regions using the same solvents. Supplementary Figure 7 illustrates the TIC and mass spectra of noncancerous kidney tissue using the $\mathrm{EtOH} / \mathrm{H}_{2} \mathrm{O}(1 / 1)$ solvent as representative for all alcohols/ $\mathrm{H}_{2} \mathrm{O}$ solvents. In the figure, the ion intensities of TAGs obtained from the noncancerous region does not increase significantly with spray time, $\mathrm{T} 1 \rightarrow \mathrm{T} 3$ in single shot PESI analysis. This proved that the main components of noncancerous kidney tissue are PCs and cholesteryl esters (CEs).

\section{Comparison of Two Modes of Measurements- Single and Continuous Shot}

The single shot experiment is suitable for measurements of time-dependent PESI mass spectra in $20 \mathrm{~s} \mathrm{[10].} \mathrm{By} \mathrm{this} \mathrm{mode,}$ the needle was replaced every time for different tissue samples. When we measured the same tissue at different regions, the needle was rinsed by $50 \%$ acetone and water every time to avoid the cross contamination. The single-shot mode using a $0.5 \mathrm{~mm}$-diameter $\mathrm{Ti}$ needle reflected changes in emerging spectra as a function of time because electrospray continued for $\sim 20 \mathrm{~s}$. Therefore, the single-shot mode could be quite useful for resolving the time window when specific mass spectra emerge.

The mass spectra obtained by single-shot PESI summed up for the total spray time were found to be basically the same as those obtained by the continuous shot mode, as expected. The measurement of time-dependent PESI mass spectra in continuous mode PESI was difficult because the duration of the electrospray in this mode was only a few hundred milliseconds. Supplementary Figure $8(\mathrm{a}-\mathrm{d})$ shows the mass spectra for the tissue that is composed of noncancerous (reddish) and cancerous (whitish) parts in the renal tissue, measured by the continuous shot mode obtained by using the binary solvent of $\mathrm{EtOH} / \mathrm{H}_{2} \mathrm{O}$. As shown in the mass spectra obtained for white parts in regions 1,2, and 3 (all of which correspond to the number depicted in inset e), ion signals due to PCs, CEs, and TAGs were detected. In contrast, in the mass spectra obtained from region 4 (reddish region), PCs and CEs were dominant ions, but TAGs were hardly detected.

\section{Conclusion}

Although it is widely known that TAGs are hallmarks of RCC, our findings indicated that TAG with different fatty acyl substituents that are not observed in noncancerous tissue could be useful for the application of PESI-MS to cancer diagnosis, as well as to metabolomics underlying the disease [22]. Additionally, sequential and exhaustive ionization of analytes by PESI-MS would guarantee significant potential for the application of mass spectrometry to rapid and real-time clinical diagnosis to guide surgery.
However, imaging with PESI-MS may damage tissue morphology and may probably be useless for further evaluation using other conventional histopathologic techniques. Extensive work is underway to expedite PESI-MS for the analysis of biological specimens with minimal morphologic destruction.

\section{Acknowledgments}

The authors acknowledge the financial support for this work by the Development of System and Technology for Advanced Measurement and Analysis Program (SENTAN) from Japan Science and Technology Agency (JST).

\section{References}

1. Nishida, T., Miwa, H., Yamamoto, M., Koga, T., Yao, T.: Bile acid absorption kinetics in Crohn's disease on elemental diet after oral administration of a stable-isotope tracer with chenodeoxycholic-11, 12-d2 acid. Gut 23, 751-757 (1982)

2. Fenn, J., Mann, M., Meng, C., Wong, S., Whitehouse, C.: Electrospray ionization for mass spectrometry of large biomolecules. Science 246, 64-71 (1989)

3. Tanaka, K., Ido, Y., Akita, S., Yoshida, Y., Yoshida, T. Detection of high mass molecules by laser desorption time-of-flight mass spectrometry. In Proceedings of the Second Japan-China Joint Symposium on Mass Spectrometry, Osaka, September. pp. 185-188 (1997)

4. Siuzdak, G.: The emergence of mass spectrometry in biochemical research. Proc. Natl. Acad. Sci. U.S.A. 91, 11290-11297 (1994)

5. Dill, A.L., Eberlin, L.S., Zheng, C., Costa, A.B., Ifa, D.R., Cheng, L., Masterson, T.A., Koch, M.O., Vitek, O., Cooks, R.G.: Multivariate statistical differentiation of renal cell carcinomas based on lipidomic analysis by ambient ionization imaging mass spectrometry. Anal. Bioanal. Chem. 398, 2969-2978 (2010)

6. Eberlin, L.S., Dill, A.L., Golby, A.J., Ligon, K.L., Wiseman, J.M., Cooks, R.G., Agar, N.Y.R.: Discrimination of Human Astrocytoma Subtypes by Lipid Analysis Using Desorption Electrospray Ionization Imaging Mass Spectrometry. Angew. Chem. Int. Ed. 49, 5953-5956 (2010)

7. Eberlin, L.S., Ferreira, C.R., Dill, A.L., Ifa, D.R., Cooks, R.G.: Desorption electrospray ionization mass spectrometry for lipid characterization and biological tissue imaging. Biochim. Biophys. Acta 1811, 946-960 (2011)

8. Schäfer, K., Dénes, J., Albrecht, K., Szaniszló, T., Balog, J., Skoumal, R., Katona, M., Tóth, M., Balogh, L., Takáts, Z.: In Vivo, In Situ Tissue Analysis Using Rapid Evaporative Ionization Mass Spectrometry. Angew. Chem. Int. Edit. 48, 8240-8242 (2009)

9. Hiraoka, K., Nishidate, K., Mori, K., Asakawa, D., Suzuki, S.: Development of probe electrospray using a solid needle. Rapid Commun. Mass Spectrom. 21, 3139-3144 (2007)

10. Mandal, M.K., Chen, L.C., Hiraoka, K.: Sequential and Exhaustive Ionization of Analytes with Different Surface Activity by Probe Electrospray Ionization. J. Am. Soc. Mass Spectrom. 22, 1493-1500 (2011)

11. Yoshimura, K., Chen, L.C., Asakawa, D., Hiraoka, K., Takeda, S.: Physical properties of the probe electrospray ionization (PESI) needle applied to the biological samples. J. Mass Spectrom. 44, 978-985 (2009)

12. Chen, L.C., Yoshimura, K., Yu, Z., Iwata, R., Ito, H., Suzuki, H., Mori, K., Ariyada, O., Takeda, S., Kubota, T., Hiraoka, K.: Ambient imaging mass spectrometry by electrospray ionization using solid needle as sampling probe. J. Mass Spectrom. 44, 1469-1477 (2009)

13. Yoshimura, K., Chen, L.C., Yu, Z., Hiraoka, K., Takeda, S.: Real-time analysis of living animals by electrospray ionization mass spectrometry. Anal. Biochem. 417, 195-201 (2011)

14. Righi, V., Mucci, A., Schenetti, L., Tosi, M.R., Grigioni, W.F., Corti, B., Bertaccini, A., Franceschelli, A., Sanguedolce, F., Schiavina, R., Martorana, G., Tugnoli, V.: Ex vivo HR-MAS magnetic resonance spectroscopy of normal and malignant human renal tissues. Anticancer Res. 27, 3195-3204 (2007)

15. Cohen, H.T., McGovern, F.J.: Medical Progress Renal-Cell Carcinoma. N. Engl. J. Med. 353, 2477-2490 (2005)

16. Mandal, M.K., Chen, L.C., Yu, Z., Nonami, H., Erra-Balsells, R., Hiraoka, K.: Detection of protein from detergent solutions by probe electrospray ionization mass spectrometry (PESI-MS). J. Mass Spectrom. 46, 967-975 (2011) 
17. Shanta, S.R., Zhou, L.-H., Park, Y.S., Kim, Y.H., Kim, Y., Kim, K.P.: Binary matrix for MALDI imaging mass spectrometry of phospholipids in both ion modes. Anal. Chem. 83, 1252-1259 (2011)

18. Goodwin, R.J.A., Pennington, S.R., Pitt, A.R.: Protein and peptides in pictures: imaging with MALDI mass spectrometry. Proteomics 8, 3785-3800 (2008)

19. MacAleese, L., Stauber, J., Heeren, R.M.A.: Perspectives for imaging mass spectrometry in the proteomics landscape. Proteomics 9, 819-834 (2009)

20. Seeley, E.H., Oppenheimer, S.R., Mi, D., Chaurand, P., Caprioli, R.M.: Enhancement of protein sensitivity for MALDI imaging mass spec- trometry after chemical treatment of tissue sections. J. Am. Soc. Mass Spectrom. 19, 1069-1077 (2008)

21. Eberlin, L.S., Ferreira, C.R., Dill, A.L., Ifa, D.R., Cheng, L., Cooks, R.G.: Nondestructive, Histologically Compatible Tissue Imaging by Desorption Electrospray Ionization Mass Spectrometry. Chem. Bio. Chem. 12, 2129-2132 (2011)

22. McAnoy, A.M., Wu, C.C., Murphy, R.C.: Direct qualitative analysis of triacylglycerols by electrospray mass spectrometry using a linear ion trap. J. Am. Soc. Mass Spectrom. 16, 1498-1509 (2005) 